\title{
Synthesizing CdSe nanoparticles by using a low concentration of cadmium ions and the apoferritin protein cage of marine pennate diatoms
}

\author{
Kenji Iwahori* ${ }^{1}$, Midori Yamane ${ }^{2}$, Sakiko Fujita ${ }^{2}$, and Ichiro Yamashita ${ }^{2,3}$ \\ 1, PRESTO, Japan Science and Technology Agency, 4-1-8 Honcho, Kawaguchi, Saitama 332-0012, Japan \\ 2, Graduate School of Materials Science, Nara Institute of Science and Technology, 8916-5, Takayama, \\ Ikoma, Nara 630-0101, Japan \\ 3, Graduate School of Engineering, Osaka University, 8-1 Mihogaoka, Ibaraki, Osaka 567-0047, Japan
}

Author's E-mail address ;

Kenji Iwahori ; holy@ms.naist.jp

Midori Yamane ; myamane@ms.naist.jp

Sakiko Fujita ; sakiko@ms.naist.jp

Ichiro Yamashita ; ichiro@ms.naist.jp

*Corresponding author: Kenji Iwahori

Contact and Present address;

Graduate School of Materials Science E201, Nara Institute of Science and Technology, 8916-5, Takayama, Ikoma, Nara 630-0101, Japan

E-mail: holy@ms.naist.jp

Tel: +81-743-72-6196 Fax: +81-743-72-6196

\begin{abstract}
Cadmium selenide (CdSe) nanoparticles (NPs) were first synthesized in the cavity of recombinant cage-shaped protein from marine pennate diatoms. Our constructed recombinant apoferritin (FerA-dCys) from the diatoms revealed that the removal of cysteine residues on the surface of the recombinant apoferritin enhanced the CdSe NPs synthesis in the apoferritin cavity. The CdSe NPs were synthesized in the FerA-dCys with a very low concentration of cadmium ions $(0.02 \mathrm{mM})$. The core formation ratio of CdSe NPs showed more than $20 \%$. The average diameter of synthesized CdSe NPs was $7 \mathrm{~nm}$ with a small size distribution. The synthesized NPs were analyzed by energy-dispersive X-ray spectroscopy (EDX) and high-resolution transmission electron microscopy (HR-TEM), and it was
\end{abstract}


confirmed that the synthesized NPs in the FerA-dCys were consistent with $\mathrm{Cd}$ and Se ions.

\section{Keywords}

ferritin, cadmium selenide, nanoparticle, biomaterials, compound semiconductors,

\section{Main TEXT}

\section{Introduction}

Cadmium pollutants in industrial waste and the drainage of mines are a large problem in the natural environment. Many countries have established strict environmental standards for limiting cadmium ion levels in soil and drainage. Many methods to eliminate cadmium ions in environmental waste have been proposed by numerous researchers $[1,2]$. However, one of the most important problems from a cost-savings and technical perspective is the elimination of very low concentrations of cadmium ions in the large quantity of polluted drainages. On the other hand, cadmium ions are essential resources for the production of various electrical devices. For instance, as II-VI compound semiconductor NPs, cadmium selenide (CdSe) nanoparticles (NPs) are used for solar cells, lights, various sensors, etc. Since the size and stoichiometry of CdSe NPs affect their band gap and photoluminescence characteristics, the controlled synthesis of CdSe NPs is indispensable for many electric devices [3].

Fabricating NPs by using bio-templates is an effective approach for the controlled synthesis of NPs with various sizes and morphologies. A lot of efforts have been made to make a wide variety of NPs by utilizing diverse kinds of bio-templates, such as proteins or viruses. One of the bio-templates, apoferritin protein from eukaryotes such as mammals, plants, and algae, is usually a spherical supra-molecular protein with a diameter of $12 \mathrm{~nm}$ and a cavity of $7 \mathrm{~nm}$ [4]. The protein shell, as a restricted nano-mold, is composed of 24 polypeptide subunits with eight channels through which ions can enter the cavity and form homogeneous NP cores with a diameter of $7 \mathrm{~nm}$. We have already fabricated not only many kinds of metals oxides but also various semiconductor NPs such as CdS [5, 6], CdSe [7, 8], ZnSe [9], $\mathrm{Au}_{2} \mathrm{~S}[10]$, $\mathrm{CuS}[11]$ in a horse spleen apoferritin (HsAFr) cavity by using a slow chemical reaction system (SCRY). The synthesis of the above semiconductor NPs were achieved using high concentrated and positively charged metal ions (upper $1 \mathrm{mM}$ ) because the NPs could not be observed by using a low concentration of positively charged ions in the cavity [5-11].

Recently, a ferritin from a marine pennate diatom, Pseudo-nitzschia multiseries, which lives in iron-limiting coastal ocean was found. This ferritin has a spherical protein shell with a diameter of $12 \mathrm{~nm}$ and an inner cavity, the same as the HsAFr. It accumulates iron oxide mineral into the inner cavity under iron-limiting area. The gene expression of this ferritin was affected by the concentration of iron ions in 
the environment. As a result, diatoms having ferritin protein can survive under unfavourable concentrations of iron ions [12]. In the present work, we constructed some apoferritin derivatives from the marine pennate diatoms and succeeded in synthesizing homogenous semiconductor CdSe NPs in the cavity at a low concentration of cadmium ions instead of iron ions. The presumable bio-mineralization mechanisms that will provide valuable knowledge about the low concentration of positively charged ions are also discussed.

\section{Material and methods}

We constructed a recombinant apoferritin, FerA. The DNA sequence of the FerA from the marine pennate diatoms, Pseudo-nitzschia multiseries [12], was referred to the GenBank (Accession: FJ004953.1). A whole DNA fragment of the ferritin was synthesized, and it was cloned to a plasmid (pET30a). The plasmid was then transformed to E. Coli (BL21(DE3)) as a host cell. The cells were cultured in $3 \mathrm{~L}$ of an LB medium that included ampicillin $(100 \mu \mathrm{g} / \mathrm{mL})$ at $26^{\circ} \mathrm{C}$ for $16 \mathrm{~h}$. In addition, the FerA-dCys recombinant apoferritin, in which two cysteine of 77th and 111th of a FerA subunit (77-cys and 111-cys) were replaced with alanine residues, was constructed by a typical site-specific mutagenesis [9]. All of the recombinant apoferritins were purified by the previously described method using column chromatography [9]. The apoferritin from horse spleen (HsAFr) was purchased from SIGMA-Aldrich (A3641). Dynamic light scattering (DLS) measurement was conducted to observe the precipitation of each apoferrtin, HsAFr, FerA, and FerA-dCys. The $200 \mu \mathrm{L}$ of reaction solution with each apoferritin and $0-0.5 \mathrm{mM}$ cadmium acetate were mixed into the cuvette. The detailed method was described in a reference [13].

CdSe NP cores were synthesized with a basic reaction solution (total volume of $3 \mathrm{~mL}$ or $0.3 \mathrm{~mL}$ ) including $0.1 \mathrm{mg} / \mathrm{mL}$ of each apoferritin, $40 \mathrm{mM}$ ammonia acetate, $5 \mathrm{mM}$ ammonia water, $5 \mathrm{mM}$ selenourea, and $0-1.0 \mathrm{mM}$ cadmium acetate for overnight incubation at $25^{\circ} \mathrm{C}$ and $\mathrm{pH} 8.0$ [7]. After the overnight incubation, the reaction solutions were centrifuged at $10,000 \mathrm{rpm}$ for 1 minute to remove the large bulk precipitation. Then $4 \mathrm{uL}$ of supernatant was put on the carbon grid and stained with $1 \%$ aurothioglucose, which does not stain the apoferritin cavity. The samples were observed by transmission electron microscopy (TEM; JEM-2200FS, JEOL). To analyze the synthesized CdSe NPs in the apoferritin cavity by EDX, after overnight incubation the reaction solutions were concentrated and washed with deionised water by using an ultrafiltration membrane (Amicon, GE 50,000 cut off). Free cadmium ions and selenium ions in the reaction solution were removed by this treatment. This step was repeated 4 or 5 times. 
A

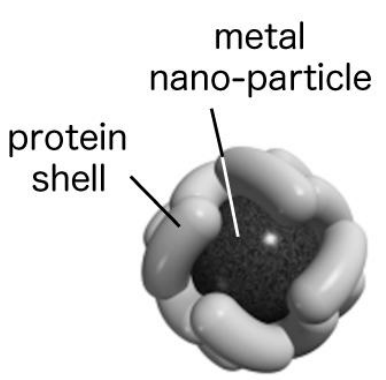

B

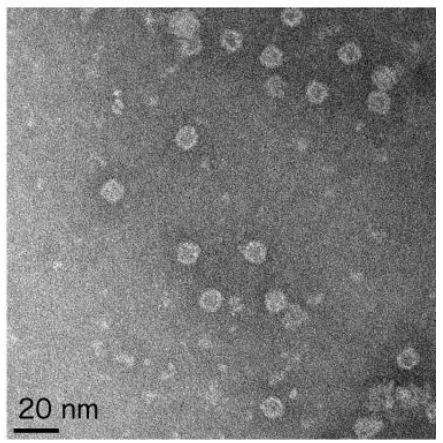

Figure 1, Model of apoferritin composed of 24 subunits such as FerA and FerA-dCys apoferritin (A), and TEM image of purified recombinant apoferritin, FerA-dCys (B). Diameter of FerA-dCys is $12 \mathrm{~nm}$ with $7 \mathrm{~nm}$ inner cavity diameters. TEM image was stained by $1 \%$ phosphotungstic acid. Bar represents $20 \mathrm{~nm}$.

\section{Results and Discussion}

We constructed and purified the recombinant apoferritins FerA and FerA-dCys. Fe oxide NPs were synthesized in the cavity of each purified apoferritin (data not shown), suggesting that these recombinant apoferritins can function normally. Our previous studies reported the success of CdSe NPs synthesis in the horse spleen apoferritin (HsAFr) by the basic reaction solution including $1 \mathrm{mM}$ cadmium acetate (see Material and Methods), and the core formation ratio (CFR) of CdSe NPs was in the upper 80\% range [7, 8]. First, we synthesized CdSe NPs in the FerA apoferritin using the same basic reaction solution including $1 \mathrm{mM}$ cadmium acetate. However, the CdSe NP cores were not observed in the FerA apoferritin cavity by TEM analysis because many FerA apoferritins quickly gathered and were precipitated by the addition of cadmium acetate. The FerA apoferritin has two cysteine residues (111-Cys and 77-Cys) on the surface of ferritin protein. We concluded that these cysteine residues affected this gathering and precipitation. Therefore, we also constructed the other

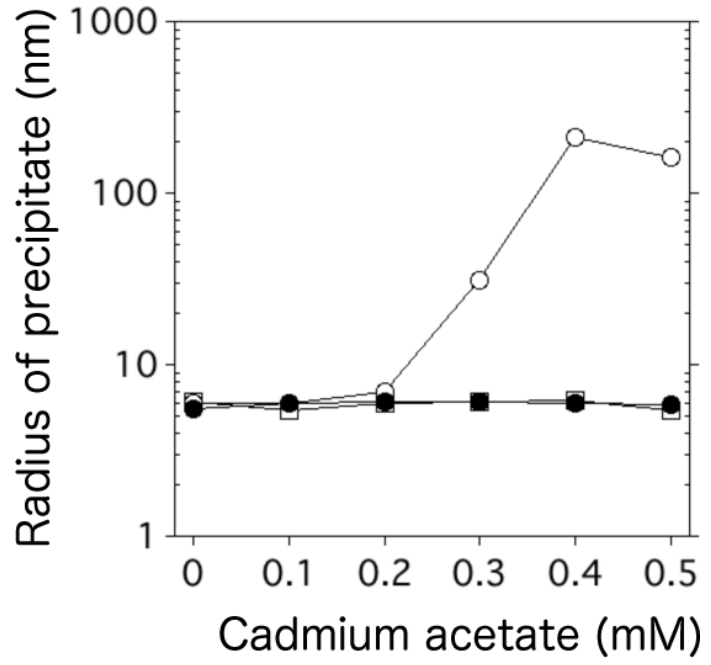

Figure 2, Effect of cadmium ions on aggregation of each apoferritin, HsAFr $(\square)$, FerA ( $\bigcirc)$, and FerA-dCys (O) by DLS measurement. recombinant apoferritin, FerA-dCys, by using a typical site-directed mutagenesis method [9]. The FerA-dCys, which replaces 111-Cys and 77-Cys with alanine amino acid, was observed by TEM as 
being round and having a $12 \mathrm{~nm}$ diameter. It also had an inner cavity with a $7 \mathrm{~nm}$ diameter (Figure 1B).

Figure 2 shows the effect of cadmium ions on the aggregation of three kinds of apoferritins. The aggregation was measured by the dynamic light scattering (DLS). The results of measuring FerA or HsAFr with cadmium ions from 0 to $0.5 \mathrm{mM}$ revealed a radius of around $6 \mathrm{~nm}$. The diameters of these apoferritins were about $12 \mathrm{~nm}$. A $6 \mathrm{~nm}$ radius strongly suggests that the ferritin proteins existed as one molecule; namely, these two kinds of apoferritins do not aggregate. In the FerA apoferritin, the aggregation was formed by the concentration of cadmium ions at more than $0.3 \mathrm{mM}$. However, the FerA-dCys apoferritin could not form the aggregation with the same concentrations of cadmium ions. The removal of two cysteine amino acid residues on the surface of the FerA apoferritin effectively avoids the aggregation caused by the addition of cadmium ions.

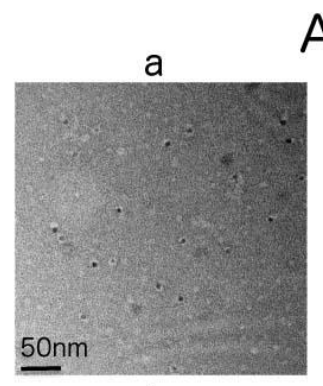

$\mathrm{b}$

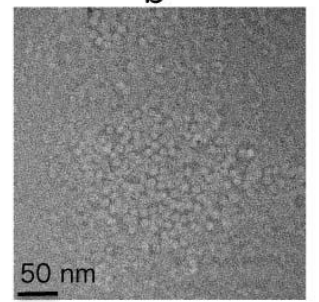

A

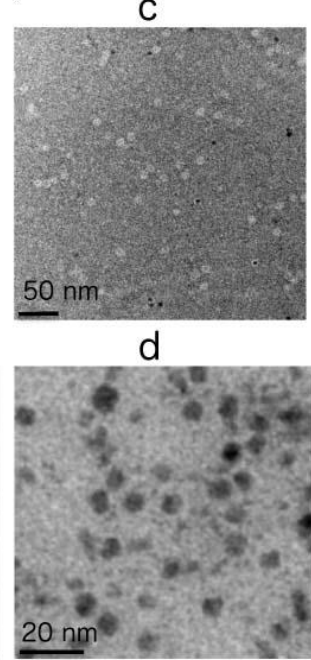

B

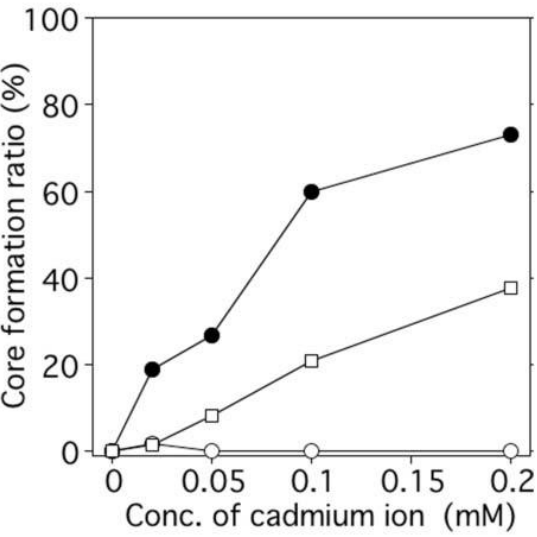

Figure 3, TEM images of CdSe NPs synthesized in three kinds of apoferritin (A): FerA-dCys (a) and FerA (b), with HsAFr (c) used as a control. All CdSe NPs were synthesized in basic reaction solution with $0.1 \mathrm{mM}$ cadmium ions. These three kinds of TEM images (a, b, c) were stained by $1 \%$ aurothioglucose. Image (d) is a TEM image of CdSe NPs synthesized in FerA-dCys without stain by $1 \%$ aurothioglucose. The reaction condition was identical to (a). Only CdSe NP cores can be observed. Core formation ratios (CFRs) of CdSe NPs synthesized in three kinds of apoferritins are shown in (B): FerA $(\bigcirc)$, FerA-dCys $(\boldsymbol{O})$, and HsAFr $(\square)$. CFRs were calculated from each TEM image [9].

We synthesized CdSe NPs in the cavity of three kinds of apoferritins with the basic reaction solution that included $0.1 \mathrm{mM}$ cadmium ions instead of $1 \mathrm{mM}$ cadmium ions (Figure $3 \mathrm{~A}$ ). Many NPs were observed in the FerA-dCys apoferritin cavity by the TEM observation (Figure 3A(a)). On the other hand, the NPs could not be synthesized in the FerA apoferritin cavity (Figure 3A(b)). However, we observed small aggregations outside of FerA apoferritins. It is likely that most free cadmium ions in the reaction solution were consumed to fabricate these small aggregations. A few NPs were synthesized in the HsAFr (Figure 3A(c)). The synthesized NPs in the FerA-dCys were observed by the TEM without 
stain. The diameter of the NPs is about $7 \mathrm{~nm}$ on the average (Figure 3A(d)). Figure 3B shows the core formation ratios (CFRs) of CdSe NPs synthesized in the three kinds of apoferritins with the basic reaction solutions that included $0,0.02,0.05,0.1$, or $0.2 \mathrm{mM}$ cadmium ions. All CFRs of FerA-dCys are higher than those of FerA and HsAFr. The highest CFR for CdSe NPs in FerA-dCys was $75 \%$ when the concentration of cadmium ions was at $0.2 \mathrm{mM}$ (Figure 3B). In addition, the CFR of NPs synthesized in the FerA-dCys apoferritin with the $0.02 \mathrm{mM}$ cadmium acetate was $20 \%$. The FerA-dCys apoferritin can fabricate the CdSe NPs with a very low concentration of cadmium ions. At the same concentration of cadmium ions, the CFR of FerA and HsAFr are very low at only $2 \%$ and $5 \%$. It is likely that the low concentration of cadmium ions can be introduced into the FerA-dCys cavity in the same manner as the low concentration of iron ions. There were many FerA-dCys ferritins filled with CdSe NPs and some vacant FerA-dCys apoferritins as shown in Figure 3A(a). From the results, it is clear that the pattern of CdSe NPs core fabrication in the FerA-dCys is an all-or-nothing type of synthesis. This process is analogous to the synthesis of iron oxide, CdSe and ZnSe NPs in the HsAFr [5, 6, 9]. There is a possibility that many kinds of NPs can be synthesized in the FerA-dCys by using different kinds of positively charged ions.

The components of the synthesized NPs in the FerA-dCys apoferritin were clarified by using TEM-EDX analysis. We prepared the CdSe NPs synthesized in the FerA-dCys with the basic reaction

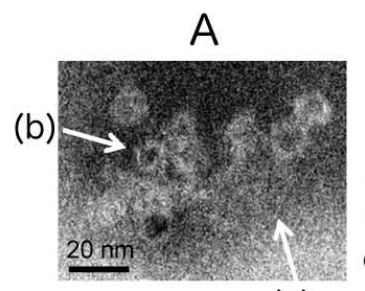

(c)
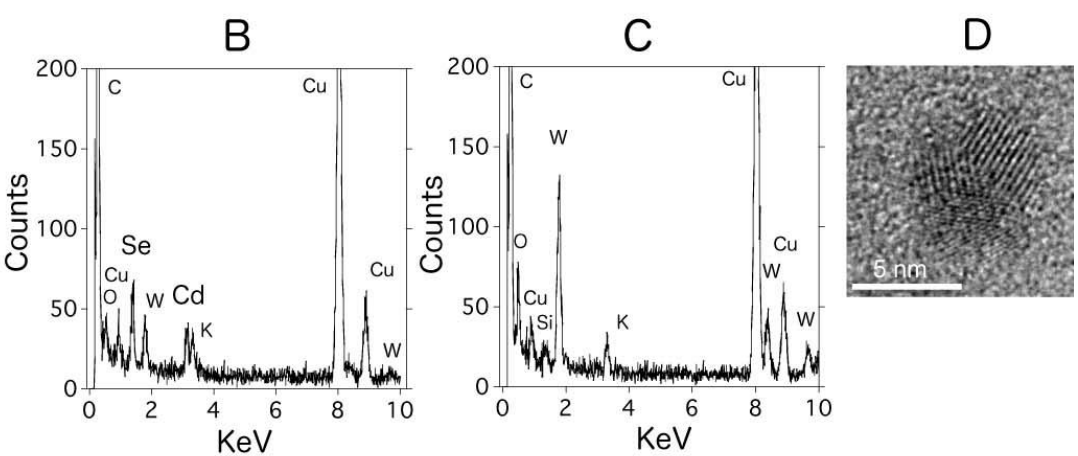

Figure 4, TEM-EDX spectra of synthesized NPs in FerA-dCys apoferritin. Two arrows in TEM image (b) and (c) in Figure A show areas of EDX analysis on TEM grid with a synthesized NP (Figure B) and on TEM grid without synthesized NPs (Figure C). In EDX results (Figure B) and (Figure C), potassium (3.31 keV) and some tungstaten $(1.78,8.40$ and $9.67 \mathrm{keV})$ come from staining reagent for TEM observation. Copper peaks $(0.93,8.05$, and 8.90 $\mathrm{keV})$, oxygen peak $(0.53 \mathrm{keV})$, carbon peak $(0.28 \mathrm{keV})$ and silicon peak $(1.74 \mathrm{keV})$ are attributed to TEM grid and protein shells. Diameter of beam for TEM-EDX analysis is about $5 \mathrm{~nm}$. Figure 4D shows the crystal lattice of a CdSe NP in the FerA-dCys synthesized by using the reaction solution with $0.1 \mathrm{mM}$ cadmium ions. This image is not stained by the aurothioglucose. The bar is $5 \mathrm{~nm}$.

solution that included $0.1 \mathrm{mM}$ cadmium acetate. Figure 4A shows the TEM image of the purified CdSe NPs in the FerA-dCys. Two arrows of (b) and (c) in Figure A show areas of EDX analysis on TEM grid with a synthesized NP (Figure 4B) and on TEM grid without synthesized NPs (Figure 4C). The EDX 
analysis of a NP in Figure 4B shows the $\mathrm{L} \alpha$ Selenium (Se) peak $(1.38 \mathrm{KeV})$ and the $\mathrm{L} \alpha$ of Cadmium (Cd) peak $(3.13 \mathrm{KeV})$. There are no peaks of Se and $\mathrm{Cd}$ in the Figure 4C. The peaks of Copper $(\mathrm{Cu})$, carbon $(\mathrm{C})$, oxygen $(\mathrm{O})$ and silicon $(\mathrm{Si})$ are attributed to the TEM grid or protein shells and the peaks of tungsten (W) are also attributed to the reagent for the stain used to observe ferritin proteins by TEM. The composition ratio of $\mathrm{Cd}$ and $\mathrm{Se}$ was estimated to be $1: 1$ (Figure 4B). These results mean that the synthesized NP is a CdSe compound [7, 8]. The crystal lattices of CdSe NPs in the FerA-dCys apoferritin were observed by the high-resolution TEM (Figure 4D). There are some domains of lattice fringes in a CdSe NP. This is indicating that the synthesized CdSe NPs are polycrystalline [7].

\section{Conclusions}

We first succeeded in fabricating CdSe semiconductor NPs in a recombinant apoferritin from the diatom (FerA-dCys) and controlled the size of the NPs in the cavity. The FerA-dCys apoferritin has a special ability to synthesize CdSe NPs at low concentration cadmium ions. Two cysteine residues per one subunit on surface of apoferritin from the diatom (FerA) prevented the introduction of low concentration cadmium ions into the FerA apoferritin cavity. Therefore, the removal of the cysteine residues from the surface of the FerA apoferritin was effective for the fabrication of CdSe NP cores at under the low concentration of cadmium ions. The synthesized water-soluble and homogeneous CdSe semiconductor NPs have the potential to be used as components for nano-electric devices such as solar cells, biosensors and various markers. In addition, since the FerA-dCys can absorb a very low concentration of Cd ions, this apoferritin should contribute to bioremediation for $\mathrm{Cd}$ pollutant waste from industries or mines.

\section{Acknowledgments}

This study was supported by PRESTO, JST.

\section{References}

[1] Tabak HH, Scharp R, Burckle J, Kawahara FK, Govind R. Advances in biotreatment of acid mine drainage and biorecovery of metals: 1 . Metal precipitation for recovery and recycle. Biodegradation. 2003;14:423-36.

[2] Romero FM, Nunez L, Gutierrez ME, Armienta MA, Ceniceros-Gomez AE. Evaluation of the potential of indigenous calcareous shale for neutralization and removal of arsenic and heavy metals from acid mine drainage in the Taxco mining area, Mexico. Arch Environ Contam Toxicol. 2011;60:191-203.

[3] Murray CB, Norris DJ, Bawendi MG. Synthesis and Characterization of Nearly Monodisperse Cde $(\mathrm{E}=\mathrm{S}, \mathrm{Se}, \mathrm{Te})$ Semiconductor Nanocrystallites. J Am Chem Soc. 1993;115:8706-15.

[4] Harrison PM, Arosio P. Ferritins: Molecular properties, iron storage function and cellular regulation. Biochem. Biophys. Acta. 1996; 1275:161-203. 
[5] Iwahori K, Yamashita I. Fabrication of CdS nanoparticles in the bio-template, apoferritin cavity by a slow chemical reaction system. Proceedings of the International Conference on Nanoscience and Technology. 2007;61:492-6.

[6] Iwahori K, Yamashita I. Size-controlled one-pot synthesis of fluorescent cadmium sulfide semiconductor nanoparticles in an apoferritin cavity. Nanotechnology. 2008;19.

[7] Yamashita I, Hayashi J, Hara M. Bio-template synthesis of uniform CdSe nanoparticles using cage-shaped protein, apoferritin. Chem Lett. 2004;33:1158-9.

[8] Iwahori K, Morioka T, Yamashita I. The optimization of CdSe nanoparticles synthesis in the apoferritin cavity. Phys Status Solidi A. 2006;203:2658-61.

[9] Iwahori K, Yoshizawa K, Muraoka M, Yamashita I. Fabrication of ZnSe nanoparticles in the apoferritin cavity by designing a slow chemical reaction system. Inorg Chem. 2005;44:6393-400.

[10] Yoshizawa K, Iwahori K, Sugimoto K, Yamashita I. Fabrication of gold sulfide nanoparticles using the protein cage of apoferritin. Chem Lett. 2006;35:1192-3.

[11] Iwahori K, Takagi R, Kishimoto N, Yamashita I. A size controlled synthesis of CuS nano-particles in the protein cage, apoferritin. Mater Lett. 2011;65:3245-7.

[12] Marchetti A, Parker MS, Moccia LP, Lin EO, Arrieta AL, Ribalet F, et al. Ferritin is used for iron storage in bloom-forming marine pennate diatoms. Nature. 2009;457:467-70.

[13] Matsui T, Matsukawa N, Iwahori K, Sano KI, Shiba K, Yamashita I. Realizing a two-dimensional ordered array of ferritin molecules directly on a solid surface utilizing carbonaceous material affinity peptides. Langmuir. 2007;23:1615-8.

[14] Yamashita I, Iwahori K, Kumagai S. Ferritin in the field of nanodevices. Biochem.Biophys.Acta. 2010;1800:846-57.

\section{Figure Captions}

Figure 1, Model of apoferritin composed of 24 subunits such as FerA and FerA-dCys apoferritin (A), and TEM image of purified recombinant apoferritin, FerA-dCys (B). Diameter of FerA-dCys is $12 \mathrm{~nm}$ with $7 \mathrm{~nm}$.

Figure 2, Effect of cadmium ions on aggregation of each apoferritin, HsAFr ( $\square$ ), FerA (०), and FerA-dCys $(\bullet)$ by DLS measurement.

Figure 3, TEM images of CdSe NPs synthesized in three kinds of apoferritin (A): FerA-dCys (a) and FerA (b), with HsAFr (c) used as a control. All CdSe NPs were synthesized in basic reaction solution with $0.1 \mathrm{mM}$ cadmium ions. These three kinds of TEM images (a, b, c) were stained by $1 \%$ aurothioglucose. Image (d) is a TEM image of CdSe NPs synthesized in FerA-dCys without stain by $1 \%$ aurothioglucose. Reaction condition was identical to (a). Only CdSe NP cores can be observed. Core formation ratios (CFRs) of CdSe NPs synthesized in three kinds of apoferritins are shown in (B): FerA $(\bigcirc)$, FerA-dCys $(\bullet)$, and HsAFr $(\square)$. CFRs were calculated from each TEM image [9].

Figure 4, TEM-EDX spectra of synthesized NPs in FerA-dCys apoferritin. Two arrows in TEM image (b) and (c) in Figure A show areas of EDX analysis on TEM grid with a synthesized NP (Figure B) and on TEM grid without synthesized NPs (Figure C). In EDX results (Figure B) and (Figure C), potassium $(3.31 \mathrm{keV})$ and some tungstaten $(1.78,8.40$ and $9.67 \mathrm{keV})$ come from staining reagent for TEM observation. Copper peaks $(0.93,8.05$, and $8.90 \mathrm{keV})$, oxygen peak $(0.53 \mathrm{keV})$, carbon peak 
1

$(0.28 \mathrm{keV})$ and silicon peak $(1.74 \mathrm{keV})$ are attributed to TEM grid and protein shells. Diameter of beam for TEM-EDX analysis is about $5 \mathrm{~nm}$. Figure 4D shows the crystal lattice of a CdSe NP in the FerA-dCys synthesized by using the reaction solution with $0.1 \mathrm{mM}$ cadmium ions. This image is not stained by the aurothioglucose. The bar is $5 \mathrm{~nm}$. 